\title{
Orbital Non-Fermi-Liquid Behavior in Cubic Ruthenates
}

\author{
M. S. Laad, ${ }^{1, *}$ I. Bradarić, ${ }^{1,2}$ and F. V. Kusmartsev ${ }^{1, \dagger}$ \\ ${ }^{1}$ Department of Physics, Loughborough University, LE11 3TU, United Kingdom \\ ${ }^{2}$ Laboratory for Theoretical and Condensed Matter Physics, The Institute of Nuclear Sciences "Vinča”, \\ P.O. Box 522, 11001 Belgrade, Serbia
}

(Received 7 February 2006; published 4 March 2008)

\begin{abstract}
We peruse various anomalous physical responses of the cubic (ferromagnetic $\mathrm{SrRuO}_{3}$ and paramagnetic $\mathrm{CaRuO}_{3}$ ) ruthenates, such as fractional power-law conductivity, anomalous Raman line shapes, and Hall currents. We show how these exciting power-law observations are naturally described within a new, local (orbital) non-Fermi-liquid state arising from strong, multiorbital Coulomb interactions. Introducing a multiorbital, correlated model treated within the dynamical mean-field theory, we also find two distinct relaxation rates for relaxation of transport in complete agreement with experiment.
\end{abstract}

Landau Fermi liquids (LFL) are the mainstay of the conventional description of electrons in metals. Electrons occupy available quantum states up to the surface of maximum energy - the Fermi surface, well described by standard one-electron band structure. The fundamental excitations correspond to free-electron-like quasiparticles having exactly the same quantum numbers as free electrons, but with widely renormalized effective masses. Adiabatic continuation arguments then suffice for a comprehensive description of low-energy responses which are effectively described in a renormalized, effective oneelectron picture. Remarkably, the LFL state has turned out to be surprisingly robust, surviving even in many $f$-electron metals where Coulomb interactions are clearly stronger than the kinetic energy.

Discovery of cuprate superconductors, along with certain rare-earth-based metals and a host of other systems have forced the emergence of a new non-Fermi liquid (NFL) paradigm: LFL theory is unable to reconcile the wide range of anomalous physical responses exhibited by these materials. Near optimal doping, the cuprates are not described by LFL ideas [1]. The most striking manifestation of this shows up in transport experiments. In a LFL, a single transport relaxation rate, originating from electronelectron scattering, governs the temperature dependence of both the dc resistivity and magnetoresistance (or, more clearly, of the dc Hall angle [2]). In stark contrast, clearly distinct relaxation rates govern the $T$ dependence of $\rho_{x x}$ and $\rho_{x y}$ in the "normal" state of high- $T_{c}$ superconductors [3]. Clearly, such behavior must have its microscopic origin in the same microscopic scattering processes which give rise to other, equally notable anomalies in resistivity, optics, Raman scattering, thermopower, etc, [1].

The NFL behavior in high- $T_{c}$ cuprates raises several important questions. For example, is this NFL behavior unique to the cuprates? Also, is this NFL behavior limited to two-dimensional systems? While similar behavior is seen in the 1D organic metals (which are described by a Luttinger liquid), it is also observed in $\mathrm{V}_{2} \mathrm{O}_{3-y}$, a 3D oxide near the antiferromagnetic (AF) metallic boundary [4]. Such a quest is clearly of great interest, and is linked to the issue of the hitherto unanticipated, new mechanisms leading to NFL behavior. Is such NFL behavior limited to two-dimensional systems, or is it more generally driven by strong electronic correlations? This general question has received scant attention to date, and cries out for a systematic exploration.

The existence of two distinct relaxation rates has been studied using varied approaches [5]. What is common to all of them is that the one-electron Green function has no pole structure at $E_{F}$ : rather, it is characterized by a branch-cut singular behavior at low energy. This leads to a LFL quasiparticle residue $(Z)$ which vanishes on the Fermi surface. Very interestingly, the detailed manner in which it does so is different in each of the theoretical approaches: in the Anderson Luttinger liquid idea, $Z \simeq \omega^{\alpha}$, while $Z$ vanishes logarithmically in the marginal-FL idea of Varma and co-workers [6]. Clearly, this difference is important, implying as it does different $\omega$ and $T$ dependences of various physical responses [5]. The fact that $Z=0$ means that there is no adiabatic continuity connecting such an interacting system to a free Fermi gas. This makes perturbation theory in interactions impossible from the outset.

With this in mind, we study the cubic ruthenates in detail. While $\mathrm{SrRuO}_{3}$ is a ferromagnetic (FM) metal below $T_{c}=159 \mathrm{~K}$, pure $\mathrm{CaRuO}_{3}$ is a quantum paramagnet down to lowest $T$, with short-ranged $\mathrm{AF}$ correlations, as evidenced by a $\Theta_{\mathrm{CW}}<0$. A host of responses, e.g., lowenergy power-law behavior in optics [7], resistivity given by the functional form $\rho(T) \simeq \sqrt{T}$ for $T>40 \mathrm{~K}$ and $T^{1.5}$ for $T<40 \mathrm{~K}$ [8] in $\mathrm{CaRuO}_{3}$, and electronic Raman scattering intensity, that is described by the functional form $I_{R}(\omega) \simeq \sqrt{\omega}$ [9], all confirm the NFL metallic state in both ruthenates. Given the qualitative resemblance to the cuprates, where such NFL responses are well documented, $[1,5]$ it is interesting to see whether magnetotransport data are equally anomalous. Figure 1(a) shows the measured Raman intensity of $\mathrm{SrRuO}_{3}$ as a function of fre- 

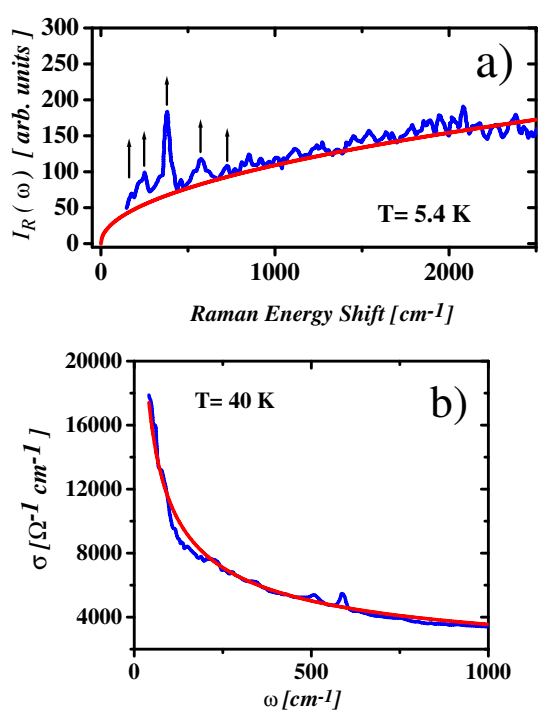

FIG. 1 (color online). (a) The electronic Raman line shape for $\mathrm{SrRuO}_{3}$ at $5.4 \mathrm{~K}$ [9] (in blue) with the fit function, $I_{R}(\omega)=$ $3.8 \sqrt{\omega}$ (solid red line). The fit is clearly visible up to $\omega_{c}=$ $3000 \mathrm{~cm}^{-1}$, and (b) The real part of the optical conductivity at $40 \mathrm{~K}$ [7] (in blue), with the fit function $\sigma(\omega)=C \omega^{-1 / 2}$ (red line).

quency (blue) [9] and a fit to the spectrum with the function $I(\omega) \sim(\omega)^{0.5}$ (red), while Fig. 1(b) shows the measured ac conductivity of $\mathrm{SrRuO}_{3}$ as a function of frequency (blue) [7] with a fit to the functional form $\sigma \sim \omega^{-0.5}$ (red). Figure 2 shows the dc Hall angle as a function of temperature (circles) [10].

In Fig. 2, clearly, $\cot \theta_{H}(T) \simeq a T^{2}$ both at high $(T>$ $40 \mathrm{~K}$ ) and low $T$, conclusively establishing the existence of two distinct relaxation rates in this system.

This is exciting: in sharp contrast to layered (lowdimensional) high- $T_{c}$ cuprates with spin $S=1 / 2$, $\mathrm{CaRuO}_{3}$ is a nearly cubic, multiorbital system with spin $S=1$ at each $\mathrm{Ru}\left(d^{4}\right)$ site. Its proximity to a possible Mott insulating phase is not obvious, nor does it show superconductivity. Closely related anomalies are found in ferromagnetic $\mathrm{SrRuO}_{3}$ much below its FM transition temperature, $T_{c}^{\mathrm{FM}}=159 \mathrm{~K}$. This compels one to search

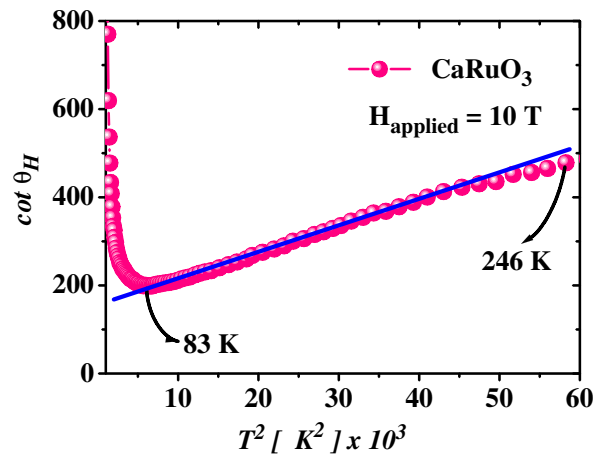

FIG. 2 (color online). The dc Hall angle, $\cot \theta_{H}\left(T^{2}\right)$ extracted from [10] for $\sigma_{x x}, \sigma_{x y}$ in $\mathrm{CaRuO}_{3}$. Similar behavior is seen for $\mathrm{SrRuO}_{3}$. for an electronic correlation origin (not related to proximity to critical magnetic fluctuations) as the key to an understanding of the NFL behavior in these cubic ruthenates. We recall that in cubic ruthenates, the $\mathrm{Ru}$ in $d^{4}$ configuration means that four $d$ electrons partially fill the threefold degenerate $t_{2 g}$ orbitals: this degeneracy is strictly preserved in the cubic structure. Considering the local orbital structure of the $\mathrm{Ru}-\mathrm{O}$ octahedron, the relevant electronically active states involve the hybridization of the triply degenerate $\mathrm{Ru}-4 d_{x y, y z, z x}$ and $\mathrm{O}-2 p_{x, y, z}$ states. In this situation, the hopping part is written as

$$
H_{\text {hop }}=-t \sum_{l \sigma}\left[d_{x y l \sigma}^{\dagger} P_{l \sigma}+\text { c.t. }+ \text { H.c. }\right] \text {, }
$$

where $\quad P_{l \sigma}=\left(p_{y, \boldsymbol{l}+\mathbf{x} / \mathbf{2}, \sigma}-p_{y, \boldsymbol{l}-\mathbf{x} / \mathbf{2}, \sigma}+p_{x, \boldsymbol{l}+\mathbf{y} / \mathbf{2}, \sigma}-\right.$ $\left.p_{x, \boldsymbol{l}-\mathbf{y} / \mathbf{2}, \sigma}\right)$ and $p_{\alpha, \boldsymbol{l} \pm \beta / 2}$ means the $p_{\alpha}$ orbital placed at the center of the bond(s) leaving site $i$ in the $\pm \beta$ direction. In Eq. (1), c.t. (cyclic terms) means terms which arise by a cyclic permutation of $x, y, z$. Notice that the $d_{x y}$ orbital does not hybridize with $p_{z, l \pm \alpha / 2}$ and $p_{\alpha, l \pm z / 2}(\alpha=x, y)$ because of symmetry reasons.

After a Fourier transform the hopping part (1), is,

$H_{\text {hop }}=-2 i t \sum_{\mathbf{k} \sigma}\left[d_{x y \mathbf{k} \sigma}^{\dagger}\left(u_{x} p_{y x \mathbf{k} \sigma}+u_{y} p_{x y \mathbf{k} \sigma}\right)+\right.$ c.t. + H.c. $]$.

Using the notations, $u_{\alpha}=\sin \left(k_{\alpha} / 2\right)(\alpha=x, y, z)$, and defining the hybridizing (Wannier) orbitals, $w_{y z \mathbf{k}}=-i\left(u_{y} p_{z y \mathbf{k}}+u_{z} p_{y z \mathbf{k}}\right) / v_{y z}, \quad w_{x z \mathbf{k}}=-i\left(u_{z} p_{x z \mathbf{k}}+\right.$ $\left.u_{x} p_{z x \mathbf{k}}\right) / v_{z x}$, and $w_{x y \mathbf{k}}=-i\left(u_{x} p_{y x \mathbf{k}}+u_{y} p_{x y \mathbf{k}}\right) / v_{x y}$. Writing $v_{\alpha \beta}=\left(u_{\alpha}^{2}+u_{\beta}^{2}\right)^{1 / 2}$ and going back to the Wannier representation we get, finally,

$$
H_{\text {hop }}=-t \sum_{\langle l m\rangle \sigma}\left[T_{y z}(m) d_{y z l \sigma}^{\dagger} w_{y z, l+m, \sigma}+\text { c.t. }+ \text { H.c. }\right]
$$

with $T_{\alpha \beta}(m)=\left\langle 2\left(u_{\alpha}^{2}+u_{\beta}^{2}\right)^{1 / 2} e^{i \mathbf{k} \cdot \mathbf{m}}\right\rangle_{\mathbf{k}}$. Thus, in the perfect cubic symmetry, the band structure is purely twodimensional in each direction solely as a consequence of the orbital orientation. This intrinsic frustration in the otherwise perfectly cubic structure leads to large quantum disorder and dynamically quenches the $t_{2 g}$ orbital order in the cubic ruthenates.

The interaction Hamiltonian in the $t_{2 g}$ sector is given by

$H_{\mathrm{int}}=U \sum_{i \alpha} n_{i d \alpha \uparrow} n_{i d \alpha \downarrow}+U^{\prime} \sum_{i, \alpha \beta}^{\prime} n_{i d \alpha} n_{i d \beta}-J_{H} \sum_{i, \alpha \beta}^{\prime} \mathbf{S}_{i d \alpha} . \mathbf{S}_{i d \beta}$

and so the full Hamiltonian is $H=H_{\text {hop }}+H_{\text {int }}$.

The peculiar feature coming from the local quantum chemistry of $\mathrm{Ca} / \mathrm{SrRuO}_{3}$ is now clear. The $d_{z \alpha}\left(w_{z \alpha}\right)$ do not hybridize with the $d_{x y},\left(w_{x y}\right)$ orbitals, but interact with a strength $U^{\prime}$, and similarly, the $d_{x y}$ do not hybridize with the $d_{y z}$, and the $d_{y z}$ do not hybridize with the $d_{z x}$. Hence, for example, the term $U^{\prime} \sum_{\langle l m\rangle \sigma} n_{l d x y \sigma} n_{l d y z \sigma^{\prime}}$ provides a nonhybridizing (screening) channel that strongly scatters the 
$d_{y z}$-band carriers. Within the impurity approximation, local non-Fermi liquid behavior [11] now arises from the competition of this term, which drives the system to a nonFL state characterized by $x$-ray edge singularities, and the $d_{\alpha \beta}-d_{\gamma \alpha}$ hybridization, which would drive the system to a FL fixed point if the nonhybridizing channel were absent. In what follows, we show that the true low-energy response is indeed power law in nature, as indicated by optical measurements.

Let us first consider the simplified situation when the screening channel is inoperative. In this case, the hybridization term drives the system to a local Fermi liquid fixed point, as is understood from the observation that $t_{p d}$ is a relevant variable [11]. This problem is then mapped onto the problem of quasiparticles hopping in an effective medium determined by a complex self-energy, $\Sigma(\omega)$, having the correct Fermi liquid form at low energy,

$$
\Sigma_{\alpha \beta}^{\mathrm{reg}}(\omega)=-\left(U^{\prime} \rho_{0}\right)^{2}\left[\frac{4 \omega}{\pi}+i \frac{\pi \rho_{0}}{2}\left(\omega^{2}+\pi^{2} T^{2}\right)\right],
$$

where $\rho_{0}$ is the band density-of-states (DOS) at the Fermi surface. So the effective model without the screening channel can be written as,

$$
H_{\mathrm{eff}}^{0}=\sum_{k \sigma \alpha \beta}\left[\epsilon_{\alpha \beta}(\mathbf{k})+\Sigma_{\alpha \beta}^{\mathrm{reg}}(\omega)\right] d_{\alpha \beta \mathbf{k} \sigma}^{\dagger} d_{\alpha \beta \mathbf{k} \sigma} .
$$

Since $\Sigma_{\alpha \beta}^{\mathrm{reg}}(\omega)$ has the FL form, we ignore it in what follows, absorbing its effect into an overall effective mass renormalization. The presence of the screening channel modifies the effective Hamiltonian, which now reads,

$$
H_{\mathrm{eff}}=H_{\mathrm{eff}}^{0}+U^{\prime} \sum_{l \mathbf{k} \mathbf{k}^{\prime}, \alpha \beta \gamma \sigma \sigma^{\prime}} n_{l d \alpha \beta \sigma} d_{\gamma \mathbf{k} \sigma^{\prime}}^{\dagger} d_{\gamma \mathbf{k}^{\prime} \sigma^{\prime}}
$$

with $\alpha \beta=x y, y z, z x$ in pairs, and $\gamma=y z$, when $\alpha \beta=$ $x y$, etc. This is recognizable as a model where the absence of the $d_{\alpha \beta}-d_{\gamma \alpha}$ hybridization results in x-ray edgelike singularity right at the Fermi surface, resulting in breakdown of FLT. We emphasize that this strong scattering of the "localized" $d_{\gamma \alpha}$ orbital results from the intrinsic feature of the quantum chemistry of $\mathrm{Ca} / \mathrm{SrRuO}_{3}$, namely, from the orbital degeneracy in the $t_{2 g}$ sector and the peculiar structure of the hybridization matrix derived above. It is not related to extrinsic disorder-induced localization.

To obtain an analytic insight into the origin of the anomalous non-FL features in the optical response, we make some simplifications which will not qualitatively modify the conclusions. The dispersive band just below the Fermi level is approximated by $\epsilon_{\alpha \beta}(k)=A-B k^{2}$ for small $k$. Furthermore, we assume that the lattice form factors are constant, since they are expected to be smooth functions of $k$. The above approximations remove the dependence of two-particle quantities on $k$ as well as on the orbital indices, and the problem is thereby transformed into that of a band of $d$ electrons scattered locally by a localized, non- hybridizing $d$ electron potential right at the Fermi surface. As is known [12], this problem is exactly soluble in the impurity limit; we work only in the metallic phase, and so choose a Lorentzian unperturbed DOS. As long as there is no band splitting, we expect this simplification to hold qualitatively. The smooth part of the $d$ electron DOS is now given by [12],

$$
\rho_{d_{\alpha \beta}}(\omega)=\frac{1-n_{d \gamma \alpha}}{\omega^{2}+D^{2}}+\frac{n_{d \gamma \alpha}}{\left(\omega-U^{\prime}\right)^{2}+D^{2}}
$$

where $D$ is the full $d$ bandwidth. The localized $d$-hole spectral function is [12]

$$
\begin{aligned}
\rho_{d \gamma \alpha}(\omega) \simeq & \left(1-n_{d \alpha \beta}\right) \frac{\theta(\omega-\mu)}{|\omega-\mu|^{1-\eta}} \\
& +n_{d \alpha \beta} \frac{\theta\left(\omega-U^{\prime}-\mu\right)}{\left|\omega-U^{\prime}-\mu\right|^{1-\eta}},
\end{aligned}
$$

where $\pi \eta=\tan ^{-1}\left(\pi \rho_{d}(0) U^{\prime}\right)$ is the scattering phase shift of the $d_{\alpha \beta}$ electrons. Here, $\rho_{d}(0)$ is the $d$-electron DOS at $E_{F}$, and equals $1 / D \pi$ for the Lorentzian band DOS. Correspondingly, the $d$ electron self-energy is also a sum of regular and singular parts: at low energies, we have $\Sigma_{d}(\omega) \simeq \Sigma_{\gamma \alpha}(\omega) \simeq|\omega-\mu|^{1-\eta}$. This implies a vanishing Landau FL quasiparticle residue, $Z=0$. Following Schotte et al. [13], and Anderson [1], the low-energy Hamiltonian can be written in terms of orbital density waves, $\rho_{\alpha}(\theta)$, localized to the various directions, $\theta$, on the Fermi surface: $H_{\mathrm{eff}}^{(0)}=2 \pi v_{F} \sum_{q, \theta} \rho_{\alpha}(q, \theta) \rho_{\alpha}(-q, \theta)$. These are operators which satisfy the commutator relation,

$$
\left[\rho_{\alpha}(q, \theta), \rho_{\alpha}\left(q^{\prime}, \theta^{\prime}\right)\right]=(q L / 2 \pi) \delta\left(\theta, \theta^{\prime}\right) \delta\left(q,-q^{\prime}\right),
$$

reminiscent of one-dimensional bosons propagating along each radial direction from the local "impurity." The important aspect now is that the term $U^{\prime} n_{d \alpha} n_{d \beta}$ simply adds a term quadratic in $\rho_{\alpha(\beta)}$ to $H_{\text {eff }}^{(0)}$ :

This yields,

$$
H=H_{\mathrm{eff}}^{(0)}+g \sum_{q, \theta, \alpha, \beta} \rho_{\alpha}(q, \theta) \rho_{\beta}(-q, \theta),
$$

which is readily rediagonalized by a Bogoliubov transformation [1], and leads to separate Fermi velocities for charge and orbital-density waves, with $v_{\mathrm{ch}}(\theta)>v_{\text {orb }}(\theta)$. This realizes a higher-dimensional scenario of chargeorbital pseudospin (fermionlike orbiton) separation, which arises via the orthogonality catastrophe (OC). It is obtained in the multiorbital model with the use of the dynamical mean-field theory (DMFT).

The optical response has two contributions. The most singular intraband contribution to $\sigma(\omega)$ results from $\rho_{d}(\omega)$ above. Indeed, by a simple scaling argument [14], we have

$$
\sigma_{\text {intra }}(\omega)=\frac{\text { const }}{(i \omega)^{1-2 \eta}} .
$$

With a plausible ratio $U^{\prime} / D \simeq 1$ [15], we obtain $\eta=1 / 4$ 
and the optical conductivity at small $\omega, \sigma_{\text {intra }}(\omega)=$ $C \omega^{-1 / 2}$ as is indeed observed experimentally [7] [see, a comparison with the data presented in Fig. 1(b)].

The above relation holds up to an upper energy cutoff of order of $D$. There is also a nonsingular contribution from the $w$-particle DOS at higher energies. It is obvious that this is very different from a FL-like description, where $\sigma(\omega) \simeq \omega^{-2}$. Further, the dc resistivity within our approximation is directly related to $\operatorname{Im} \Sigma_{d}(\omega, T)$, giving $\rho_{\mathrm{dc}}(T) \simeq$ $T^{(1-2 \alpha)}=\sqrt{T}$. Using the Shastry-Shraiman relation [16] adapted to $d=\infty$, as discussed below, we have the electronic Raman intensity, $I_{R}(\omega)=\omega \sigma^{\prime}(\omega) \simeq \sqrt{\omega}$. Finally, we have computed the effects of irradiation induced impurity scattering on $\rho_{\mathrm{dc}}$ : using the nonperturbative $T$-matrix approach [17], we find the excess resistivity, $\Delta \rho(T) \simeq$ $T^{-1 / 2}$, strongly violating Matthiessen's rule. All these are in good agreement with experimental responses found in both $\mathrm{SrRuO}_{3}$ and $\mathrm{CaRuO}_{3}$ cubic ruthenates.

Computing the magnetotransport response is more demanding. We notice that an external magnetic field couples indirectly to the orbital fluctuations via spin-orbit coupling. Given the charge-orbital pseudospin separation as above, one expects distinct relaxation times to govern the decay of charge and orbital currents. Spin-orbit interaction couples the orbital modes to the spin, and so the decay rate for orbital currents will show up in the Hall current. Following Anderson [5], the orbiton-orbiton scattering will give an orbiton decay rate, $1 / \tau_{\text {orb }}=A T^{2}$, where $A$ is related to the orbiton bandwidth (which is an effective orbital superexchange scale). There will generically be a residual constant contribution $C\left(n_{\text {imp }}\right)$, coming from orbiton-impurity scattering, and scaling with the impurity concentration. This readily leads to $\cot \theta_{H}(T) \simeq A T^{2}$, in full qualitative agreement with the form extracted earlier in the work (see, Fig. 2). We suggest that measuring the Hall angle with a varying impurity concentration (such as under radiation damage) will confirm the factor $C\left(n_{\text {imp }}\right)$; to our best knowledge, such a study has not been done.

The actual measured Fermi surface is well described by local density approximation calculations within DMFT, since the self-energies are purely local in space. A related consequence is that vertex corrections rigorously drop out from the Bethe-Salpeter equation for the computation of the conductivities [18]. Under this assumption, the ShastryShraiman relation relating the optical conductivity to the Raman response reduces to $I_{R}(\omega)=\omega \sigma_{x x}^{\prime}(\omega)$. With $\sigma_{x x}^{\prime}(\omega) \simeq \omega^{-1 / 2}$ and $I_{R}(\omega) \simeq \sqrt{\omega}$ extracted experimentally (see, Fig. 1). This relation is satisfied remarkably well in $\mathrm{SrRuO}_{3}$. Additionally, quantum oscillation measurements on $\mathrm{SrRuO}_{3}$ yield a Fermi surface very close to that found in Ref. [19]. These observations single out the DMFT as the approximation of choice to treat strong correlations in these cubic ruthenates.

In conclusion, we have carried out a detailed analysis of the highly anomalous physical responses of the cubic ruthenates. In spite of their very different structural or magnetic properties, many of their responses are strikingly similar to those found in the normal phase of the nearoptimally doped cuprates. Based on a relevant materialspecific model, we show how strong multiorbital correlations in an orbitally degenerate situation give rise to a new, orbital non-FL metal. This is shown to arise via an OC in the orbital sector within DMFT. Various anomalous features in cubic ruthenates are provided a natural explanation. We expect our analysis here to be relevant in other transition metal/rare-earth compounds having near orbital degeneracy and strong electronic correlations.

I. B. acknowledges support from the Serbian Ministry of Science, Project No. 141014, Royal Society Grant No. 2004/R3-EF, and ESF network AQDJJ.

*mukul@mpipks-dresden.mpg.de

${ }^{\dagger}$ F.Kusmartsev@lboro.ac.uk

[1] P.W. Anderson, in The Theory of High-T Superconductivity in the Cuprates (Princeton University, Princeton, NJ, 1998).

[2] P. Coleman et al., J. Phys. Condens. Matter 8, 9985 (1996).

[3] T. Chien et al., Phys. Rev. Lett. 67, 2088 (1991).

[4] T. Rosenbaum et al., Phys. Rev. B 57, R13997 (1998).

[5] E. Abrahams et al., Phys. Rev. B 68, 094502 (2003).

[6] C. M. Varma et al., Phys. Rev. Lett. 63, 1996 (1989).

[7] P. Kostic et al., Phys. Rev. Lett. 81, 2498 (1998).

[8] L. Klein et al., Phys. Rev. B 60, 1448 (1999); Y.S. Lee et al., Phys. Rev. B 66, 041104 (2002).

[9] D. Kirillov et al., Phys. Rev. B 51, 12825 (1995). We have fitted the Raman intensity of the electronic background to the form $C \cdot \sqrt{\omega}$ up to $3000 \mathrm{~cm}^{-1}$; see Fig. 1(a). The data were taken at $5.4 \mathrm{~K}$ by triple scanning spectrometer where all types of contributions with different polarizations have been included. In our view this observed background is associated with low-energy orbital excitations. At the energy higher than $3000 \mathrm{~cm}^{-1}$ the magnetic excitations may already take over the orbital ones. Therefore, this part of the spectrum has been excluded in our fits.

[10] G. Cao et al., Phys. Rev. B 56, 321 (1997); S.C. Gausepohl et al., Phys. Rev. B 54, 8996 (1996).

[11] G. M. Zhang et al., Phys. Rev. B 49, 7759 (1994).

[12] Q. Si et al., Phys. Rev. B 46, 1261 (1992).

[13] K. D. Schotte et al., Phys. Rev. 182, 479 (1969); A. Luther, ibid. 19, 320 (1979).

[14] P. W. Anderson, Phys. Rev. B 55, 11785 (1997); Phys. Rev. Lett. 67, 2092 (1991).

[15] Hyeong-Do Kim et al., Phys. Rev. Lett. 93, 126404 (2004); P. S. Cornaglia and A. Georges, Phys. Rev. B 75, 115112 (2007).

[16] B. S. Shastry et al., Phys. Rev. Lett. 65, 1068 (1990).

[17] C. M. Varma, Phys. Rev. Lett. 79, 1535 (1997).

[18] A. Khurana, Phys. Rev. Lett. 64, 1990 (1990).

[19] E. Müller-Hartmann, Z. Phys. B 76, 211 (1989); For experimental details, see A.P. MacKenzie et al., Phys. Rev. B 58, R13318 (1998). 